\title{
The Influence of the Device on User Performance in Handheld Augmented Reality
}

\author{
Silvio R. R. Sanches, Marcio A. Oizumi, Claiton Oliveira, Antonio C. Sementille and Cléber G. Corrêa
}

\begin{abstract}
Augmented Reality applications, which were known to require sophisticated hardware, can now be run on handheld devices. Although such devices provide the required computer power, the size or weight of some of them may become a barrier to user performance, mainly when the interaction is by the touch on the screen. Inserted in this context, this research consists of applying a set of subjective experiments in which volunteers use an application that runs on devices with different sizes and weights. These experiments aim to measure how much the type of handheld device influences the performance of the user in Augmented Reality applications. The main contributions of this study are (i) we concluded by subjective experiments that users with previous knowledge of how to interact with a similar AR application or a game are able to improve their performance when the device is larger and (ii) we demonstrated the age of the participant was also a good indicator of the user performance in AR applications.
\end{abstract}

Index Terms-Augmented Reality, Device, Interaction

\section{INTRODUCTION}

A UGMENTED Reality (AR) applications can run on different devices [1] and their scenes, which show real and virtual elements, can be viewed through various types of displays [2]. Non-immersive desktop applications typically rely on conventional displays [3] while more sophisticated immersive systems display their scenes, for example, on HeadMounted Displays (HMDs) [4] or on projective displays [5].

The human-computer interaction, or simply interaction, performed in AR systems, can also be of different types, varying according to the device available for this purpose [6]. The movement of fiducial markers [3] and the manipulation of virtual elements through of data gloves [7] or gesture recognition [8] are examples of ways of interacting with AR systems.

The increased computational capacity of handheld devices has made them an important platform for mobile games [9], including AR applications. The popularity of games such as Pokemon Go [10] has shown that there is room for development of many new applications for entertainment.

Although the computing power on handheld devices is smaller than on more traditional platforms [11], it is enough for the execution of many AR applications. However, the weight and the screen size used to display the scene to the user may be restrictive features for some applications.

The interaction by the touch on screen, for example, encompasses aspects such as ergonomic touch difficulty in handling due to size or weight of the device. The screen of a handheld

S. R. R. Sanches, M. A. Oizumi, C. Oliveira and C. G. Corrêa are with the Federal University of Technology - Paraná, Cornélio Procópio, PR, Brazil.

A. C. Sementille is with Universidade Estadual Paulista Júlio de Mesquita Filho, Bauru, Brazil. device is small in size compared to a desktop one. On HMDs, although the size of the display is not large, its placement close to the eyes of the user allows a wider viewing area, which does not occur on handheld devices, since the user places them away from the eyes. In addition, applications that use HMDs or desktop displays usually do not use the touch on screen as a form of interaction.

There are many researches that analyze aspects of the user's perception that influence user's performance in handheld devices [12], [13]. Among the studies on perception in AR systems, some authors have investigated, for example, the influence of the quality of the rendering on the scene understanding [14] and the depth perception among the objects of the scene viewed through different sizes of displays [15]. However, these works do not specifically address the effects of the size of screens and device weight on user performance (or on specific groups of users) when the interaction is the touch on screen, which is the most common type of interaction in AR applications. Information about the most appropriate type of device may be important, especially for users seeking the best possible performance in the application, for example, game users. The games represent a large number of the AR applications developed for handheld devices.

The objective of this research is to identify, through controlled experiments, how much the user performance is affected by the type of device. AR engineers have already studied some factors of display size, so their applications can be adjusted accordingly. However, even with the best possible adjustment, smaller screens may compromise the interaction, at least for a specific group of users (e.g. those who explore the limits of the application). Another feature to be considered is the weight, since the users handle these devices (they held and movement) for interacting with the applications.

The results shown that new users are not able to improve their performance when the device is larger. Advanced users, on the other hand, presented improvement in the performance in the accomplishment of the assigned task. In addiction, we concluded that the age of the participant was also a good indicator of the user performance.

\section{RELATED WORK}

We can find studies that analyze different aspects of the user's perception in the literature [14]. The work of Findlater and McGrenere [12], for example, shows that adaptive menus are especially important when devices with small screens are used. Raptis et al. [16] concluded that users interacting with screens larger than 4.3 inch are more efficient during information retrieval tasks. Maniar et al. [13] have demonstrated that devices equipped with larger screens can facilitate learning. 
A lot of work in literature is focused on the study of perception in AR systems, some of them are related to the device used as platform. In Kruijff et al. [14], the authors perform a research whose results can be used as a guide on perception issues in AR. Their work deals with issues such as the influence of rendering quality and pixel density for the "correct perception" of the scene.

Ghazi and Glinz [17] show in a study not related to AR that a significant number of artifacts cannot be viewed entirely, even on large screens. This means that this type of factor can influence the performance of the user interaction.

Dey et al. [15] analyzed the impact of different sizes of screens of handheld devices in relation to depth perception, which is directly related to the user performance. However, the work did not investigate the most common form of interaction in applications, the touch on screen, which encompasses aspects such as ergonomic touch difficulty in handling due to size.

Raneburger et al. [18] evaluated different Graphical User Interfaces (GUIs) adapted for smartphones and tablets. The experiment consisted of each participant performing the same task in two different layouts of the same GUI. The results pointed to some influence of screen size.

In our research, we analyzed how much the performance of one user (or a specific group of users) is affected by the type of device in AR applications that run on handheld devices. Here, we considered in our experiments the interaction by the touch on virtual objects which is the most common type of interaction in these applications.

\section{EXPERIMENTS WITH USERS}

A case study with controlled experiments was conducted, involving users and different types of devices. In the following subsections, the details on experiments are mentioned.

\section{A. Objective, Hypotheses and Assumptions}

The main objective of this work is to check how much the user performance is affected according to the type of device used in a handheld AR environment, taking into account the weight and the screen size of the device.

Two hypotheses were formulated:

- H0: the size of the display of a handheld device affects the performance of users with and without previous knowledge of how to interact with an AR application (or wich a similar aplication such as game);

- H1: we can correlate user's characteristics (height and age) with their performance in AR applications.

We defined the following assumptions for the current work:

- interaction resources, such as zoom, are not available. The size of the virtual objects cannot be modified during the experiments;

- a previous session before experiments must not be carried out. Only oral instructions must be provided for the volunteers;

- the camera of the devices must be directed to the same real object, a table, in all sessions of the experiments;

- the illumination must be the same in all sessions;

- the same fiducial marker must be used in the experiments.

\section{B. Software Apparatus}

For user performance evaluation on different screen sizes and weights, a prototype that simulates an AR application was created using Unity [19] and Vuforia [20] software. The interaction between user and prototype was defined after a search for AR applications of the most important virtual stores, Google Play and Apple Store. Table I shows the first 10 applications with the features of an AR system found on Google Play and Apple Store when the term "Augmented Reality" is searched. Applications found on Google Play were excluded from search results on Apple Store.

We identified that, in addition to moving the smartphone, the interaction by the touch on screen is the most common type in AR applications developed for handheld devices. Figure 1 shows some frames of the prototype application.

The purpose of the user in the application was to touch a virtual sphere, which was bouncing on the table (Figure 1b). The bouncing speed of the ball remained the same in all experiments. Once the user touched the sphere, the texture of the object was changed. The application has 5 steps, each of them displaying a ball textured like the ones used in some sports, such as: soccer (Figure. 1c), basketball (Figure 1d), golf, baseball and tennis. By clicking on the last sphere, the system displayed a black texture on it (Figure. 1e), finished the experiment and recorded the time taken to complete all the steps. We chose to analyze a single application in which the main type of interaction is the touch on virtual objects. This way of interacting occurs in most applications listed in the Tab. I.

\section{Hardware Apparatus}

The same prototype was executed on three handheld devices with different specifications:

- A Moto G4 plus Smartphone, 5.5-inch display, 157grams, 32GB, Octa-Core 1.5GHz processor, Android 6.0;

- A Samsung Galaxy Tablet, 7-inch display, 345grams, 8GB, Android 4.1, Dual-Core 1.0GHz processor;

- A Samsung Galaxy Tablet, 10.1-inch display, 587grams, 16GB, 1.0GHz Dual-Core processor, Android 4.1;

Although there were differences in hardware and software all of them executed the application comfortably, keeping the frame rate about thirty frames per second. Figure 2 shows the scene of the application on one of the devices used in our experiments.

\section{Volunteers and Task}

The volunteers were students, professors and administrative staff of the research institution, besides some external people. Each volunteer participated in a single application run so that a previous experiment did not facilitate their subsequent performance. One hundred forty-five people were recruited for the experiments.

The task was about interacting with the device, touching on the screen of a handheld device to select and move the virtual sphere, from an initial position to a specified target. 
Table I

TEN First APPLICATIONS WITH THE FEATURES OF AN AR SYSTEM FOUND ON GOOGLE PLAY AND the APPLE STORE WHEN THE SEARCH IS PERFORMED USING THE TERM “AugMENTED REALITY”. THE SEARCH WAS CONDUCTED ON August 6, 2018.

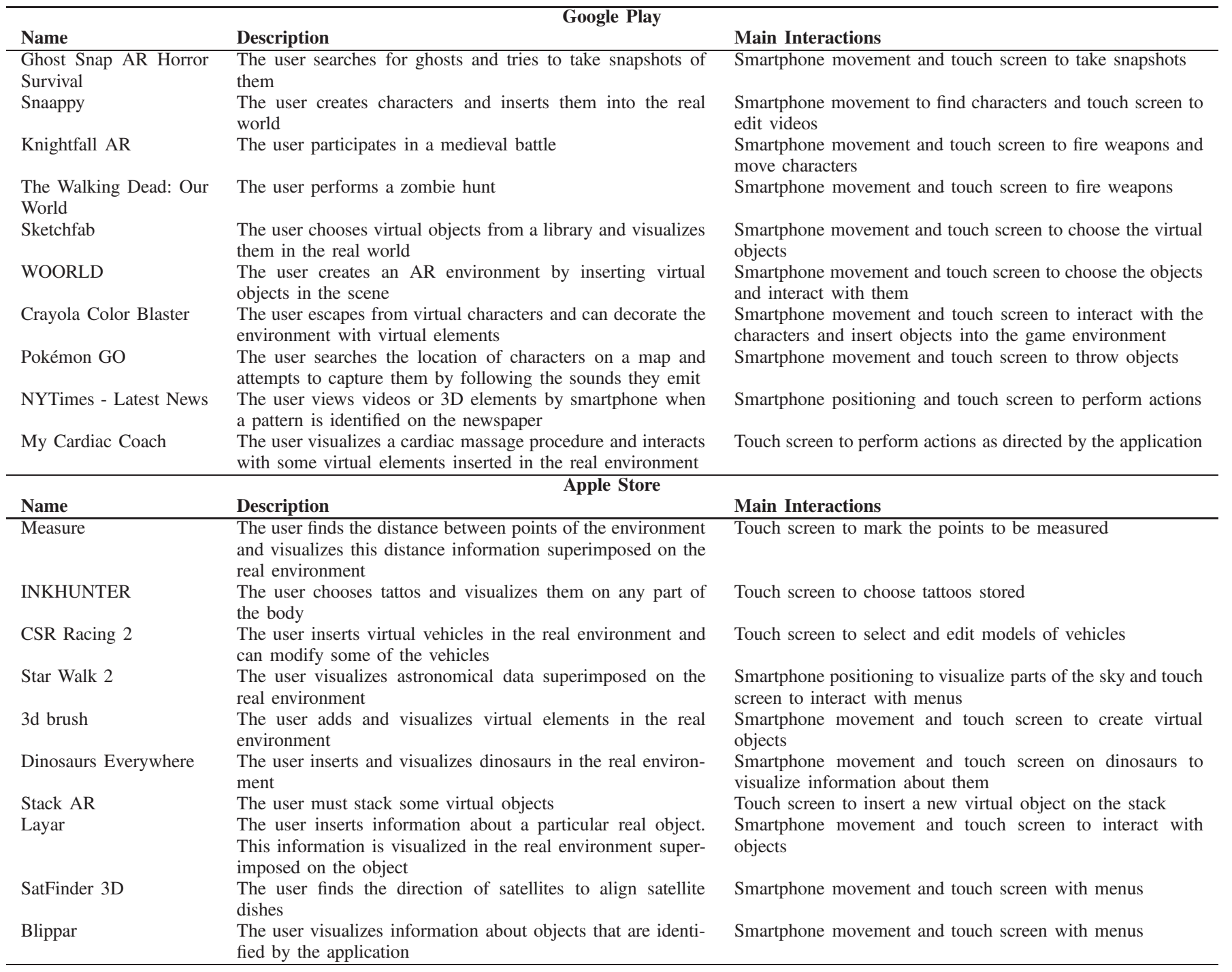

\section{E. Experimental Environment Specifications}

Since the same application was used by several volunteers, we kept the same characteristics of the testing environment in all evaluations. Details such as brightness of the environment, brightness of the screen and distance from the user to the screen followed the recommendations of the International Telecommunication Union (ITU) [21], which provides guidelines for subjective experiments in multimedia applications.

Each volunteer was instructed orally on how to proceed in the task. All were seated, arms slightly flexed, holding the device at a distance of approximately 20 centimeters $(\mathrm{cm})$ from the fiducial marker, $15 \mathrm{~cm}$ above the table and $40 \mathrm{~cm}$ between the screen and the eyes of the volunteer. The application occupied the screen in landscape mode, as a result, the device was handled the same way, held by one hand. Figure 3 shows the environment of the experiments.

The volunteers held the device as shown in Figure 3. The time spent by each volunteer for each device was measured.
The volunteers answered a post-test questionnaire, providing information about height, age, experience with mobile games and AR applications.

\section{RESUlTS}

To verify the hypothesis $\mathrm{H} 0$, we analyzed (i) if considering the data of all the users, there is correlation between the size of the display and the performance; (ii) if considering the data grouped according to the level of prior knowledge of the user in interacting with games, there is a correlation between the size of the display and the performance of the user; and (iii) if considering the data grouped according to the level of prior knowledge of the user in interacting with AR applications, there is a correlation between the size of the display and the performance of the user. First analysis aimed to show that larger devices (with larger displays) may facilitate the performance of tasks in AR systems in which the interaction is performed by touch on the screen. The number of volunteers that used the displays with sizes of 5.5, 7 and 


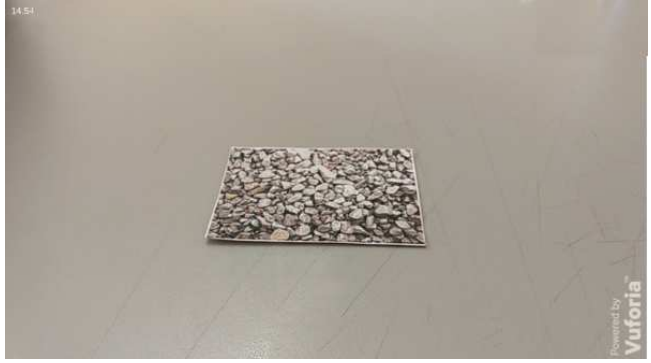

(a)

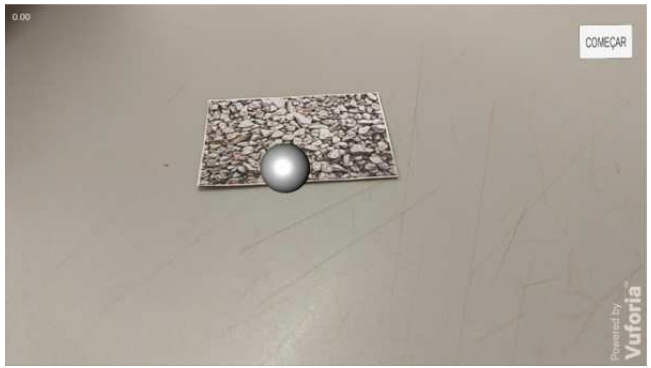

(b)

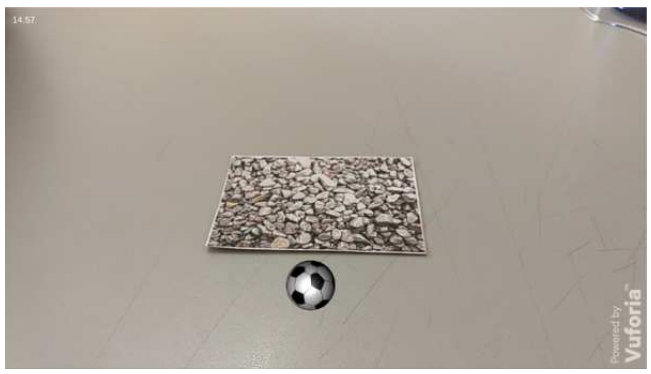

(c)

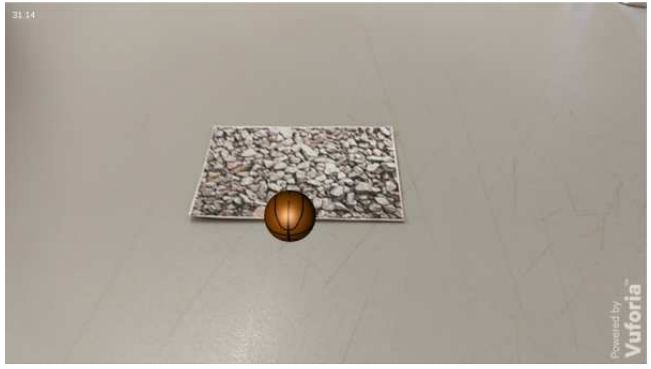

(d)

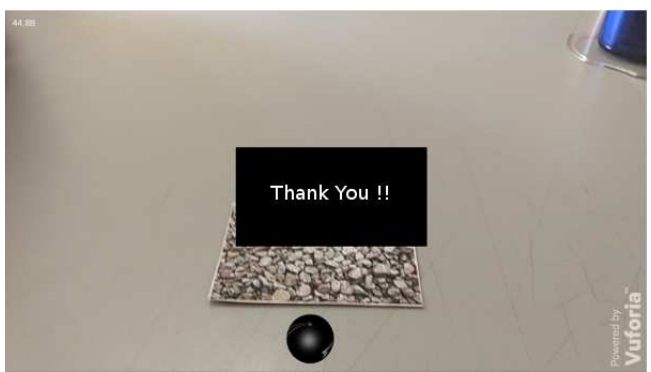

(e)

Figure 1. Some frames of the prototype application viewed by volunteers during the experiment. (a) scene without the virtual object, (b) application start screen, (c) soccer ball, (d) basketball and (e) final application screen.

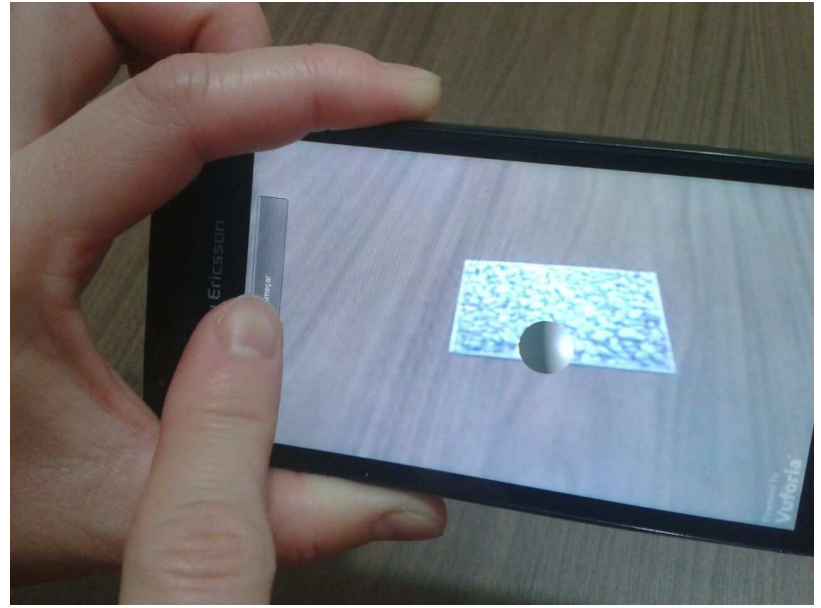

Figure 2. Scene of the application on one of the devices used in our experiments.

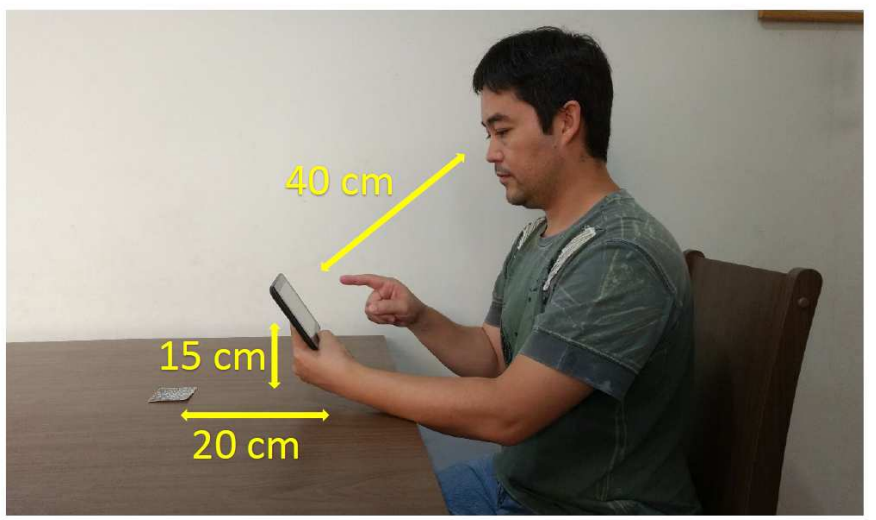

Figure 3. Environment of the experiments

10.1 inch were 56, 43 and 46 respectively. Therefore, the time spent by volunteers to perform the task was calculated for each device. Figure 4 shows the results obtained from this analysis.

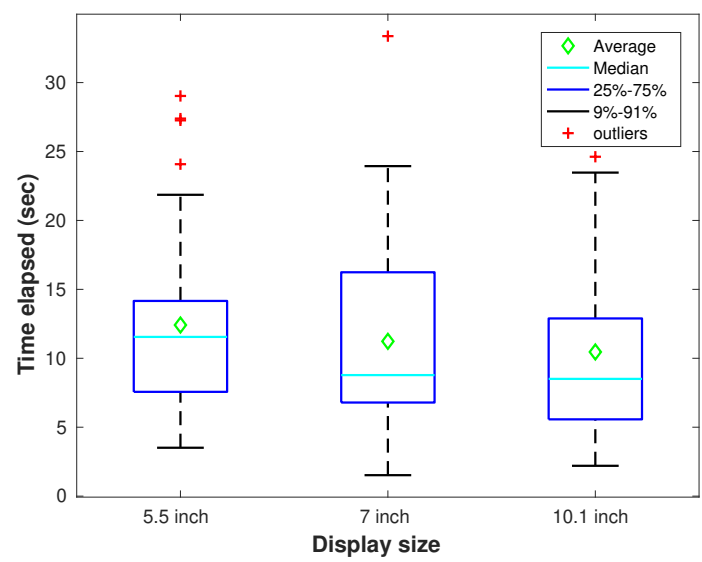

Figure 4. Times spent by volunteers using 5.5, 7 and 10.1 inch.

As it can be seen, when we considered the whole data set the average performance is best when larger displays are 
used by the handheld devices. Although some volunteers have stated that ergonomically smaller devices are easier to handle, average performance has gradually improved as the device and its display have been increased, even when the weight of the device have been increased.

The task execution times were analyzed based on different display sizes of the devices (5.5, 7 and 10.1 inch). The Shapiro-Wilk normality test was applied, showing that the data does not have normal distribution. A test that does not depend on the normal distribution of the data was used, called KruskalWallis $\mathrm{H}$ test, that also considers random independent samples and more than two samples. Each sample represents the task execution times of the volunteers for one display, resulting in three samples. The test evidenced that there is a statistically significant difference between two samples, with $p=0.0952$, i.e., $p<0.1$. A $p$-value less than 0.1 indicates that this value is statistically significant at the $90 \%$ level.

Aiming at identifying the two samples that differ between themselves, comparisons of these samples were made using Wilcoxon rank-sum test, that does not depend on the normal distribution of the data, combined with an adjustment through Bonferroni correction. The correction considered three comparisons between the three samples, and a significant value at the $90 \%$ level. The results showed a significant difference between 5.5 and 10.1 inch displays, with $p=0.087$, i.e., $p<0.1$.

The following analysis aimed to verify if the size of the display influenced on the performance of the volunteers when considering previous knowledge in the use of handheld applications with characteristics similar to the prototype (games and different AR applications).

Thus, the results of the experiments were grouped according to the following criteria: "High", the volunteer uses mobile games more than twice a week (26 volunteers); "Low", the volunteer uses mobile games less than twice a week (67 volunteers); and "None", the volunteer never used mobile games (52 volunteers). Figure 5 shows the results of this analysis.

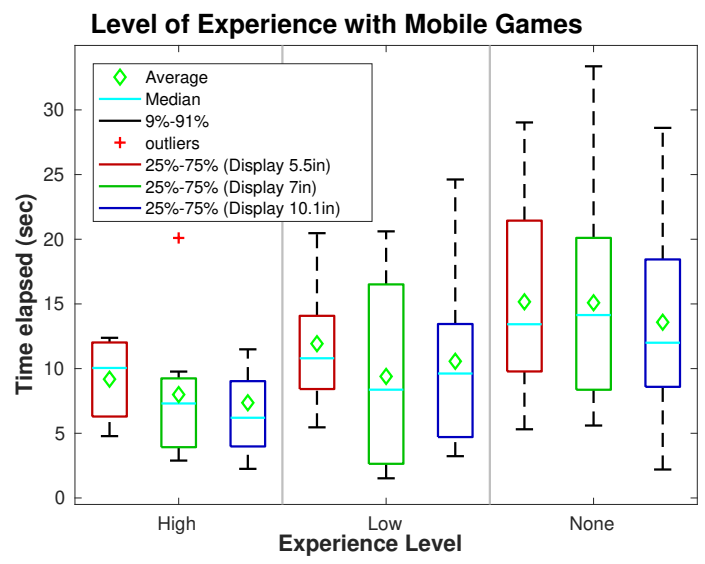

Figure 5. Results of the analysis considering groups of volunteers according to their experience with mobile games.

As it can be seen, the volunteers habituated to use mobile games have taken advantage of the device with larger display. Since they are familiar with the dynamics of interactive applications, such as games, the scenes displayed on the 10.1 inch display facilitated the performance. This is possibly due to the increased object size (sphere) that must be touched by the user, even if, for reasons of ergonomics, the larger device is difficult to handle. On the other hand, when the volunteers have no experience with mobile games, they do not take much advantage of the display size.

The following analysis is similar to the previous one, however, the results were grouped according to the level of experience of the volunteer with AR applications. The criteria used for grouping are: "High", the volunteer uses AR mobile applications (6 volunteers); "Low", the volunteer knows the AR technology and has already had some contact (32 volunteers); and "None", the volunteer does not know or has never used an AR mobile application (107 volunteers).

Some volunteers are students who have had contact with AR applications besides games with this technology. However, most of the participants are in the group "None". Figure 6 shows the results of this analysis.

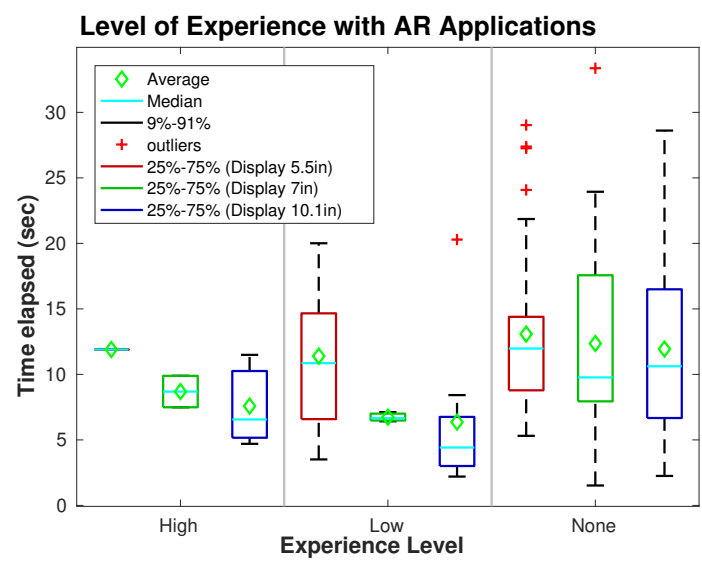

Figure 6. Results of the analysis considering groups of volunteers according to their experience with AR applications.

The results obtained in this analysis are relatively similar to the previous one. As it can be seen, users with more experience with AR applications are able to improve their performance when they use devices with larger displays.

However, statistical tests were applied to check the significant statistical differences related to user performance (task execution time) and user experience with games and AR technologies. Wilcoxon rank-sum test was used because the data does not have normal distribution and the samples are independent.

Regarding the user experience with games, we worked with two experience levels ("None"/"Low" experience and "High" experience) for the task execution times of the volunteers in each display, resulting in two samples for each display size. The test showed significant differences of performance in the:

- 5.5 inch display, with $p=0.05238$, i.e., $p \approx 0.05$;

- 7 inch display, with $p=0.0366$, i.e., $p<0.05$;

- 10.1 inch display, with $p=0.00528$, i.e., $p<0.05$. 
A $p$-value less than 0.05 indicates that this value is statistically significant at the $95 \%$ level.

Regarding the experience with AR technologies, we also worked with two experience levels ("None"/"Low" experience and "High" experience) for the task execution times of the volunteers in each display, resulting in two samples for each display size. The statistical test did not identified significant differences of performance in 5.5 and 10.1 inch displays. Significant difference was observed for performance in the 7 inch display, with $p=0.05118$, i.e., $p \approx 0.05$.

To verify the hypothesis $\mathrm{H} 1$, we analyzed (i) if there is a correlation between user height and performance, regardless of device type; (ii) if there is correlation between the age of the user and the performance of the user, regardless of the type of device; and (iii) if, considering a specific device, there is a correlation between the age of the user and the performance of the user.

Some characteristics such as the height of an user can influence the angle at which this user sees the fiducial marker. This detail can be more relevant than the type of device and, as a consequence, this angle influences his/her performance in the application. To verify the hypothesis considering the characteristic of height, the following strategy was adopted. As the volunteers used the same experimental environment, those with higher height theoretically viewed the marker less oblique than those of lower height. In other words, the angle of inclination between the device and the marker placed on the table is higher for taller people. Therefore, this peculiarity could facilitate the interaction and decrease the time spent to accomplish the task. Pearson and Spearman correlation coefficients were calculated to verify the correlation between height and performance. The results of this analysis can be seen in Figure 7.

As it can be observed, there is no correlation between the height factor and the ability to perform the task more efficiently even when we considered the data grouped according to the type of device (Figures 7b, 7c and 7d). In this way, it is understood that the angle at which the user views the marker in the scene has less influence than the type of device used.

The target audience for computer games, which are good examples of interactive applications, has long been teenagers. This reality has changed considerably in the last decade, although such applications still arouse great interest in the users of this age group.

Another characteristic to be considered in hypothesis $\mathrm{H} 1$ is the possibility that the age of the user can be used as a good measure of performance [22]. In other words, the we consider that there is a correlation between the age of the user and their performance in the application, since the visual acuity decreases significantly with age and can impair the performance of older people on devices with small displays. On the other hand, younger users tend to be more habituated to the technology in general. Therefore, factors such as experience with games, the ease of handling devices with different display sizes, for example, may be implicitly considered when comparing the age and performance of the volunteers. Figure 8 shows the results of this analysis.

As it can be observed, Figure 8 a shows that there is a

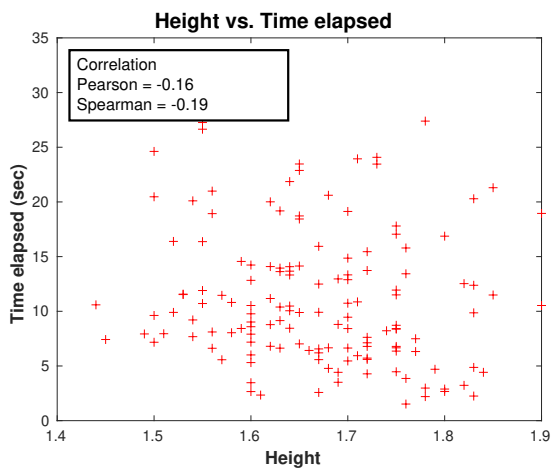

(a)

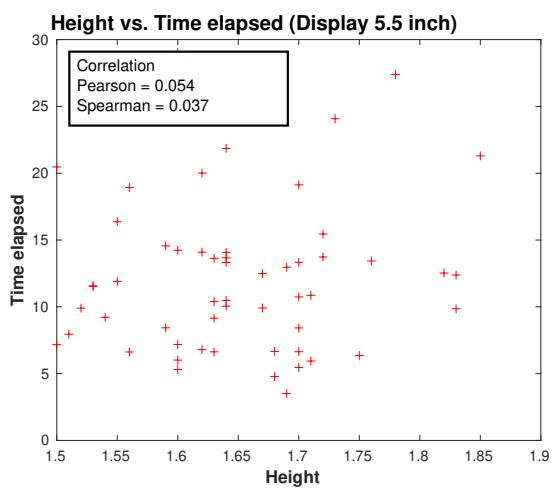

(b)

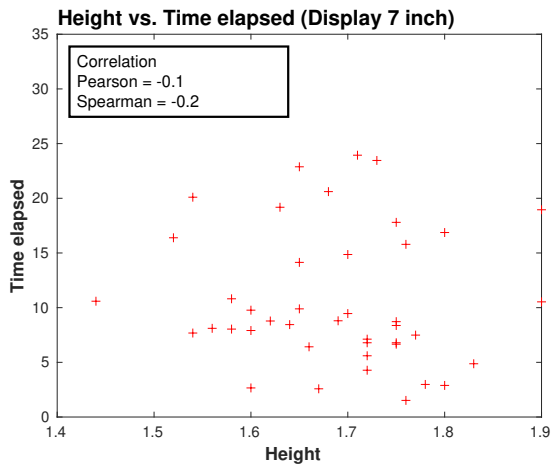

(c)

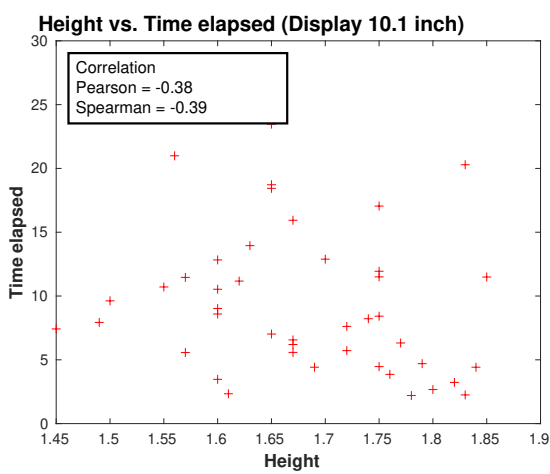

(d)

Figure 7. Correlation between the height of the volunteers and their performance. (a) data from all volunteers, (b) data from volunteers that used a 5.5 inch display, (c) data from volunteers that used a 7 inch display and (c) data from volunteers that used a 10.1 inch display. 


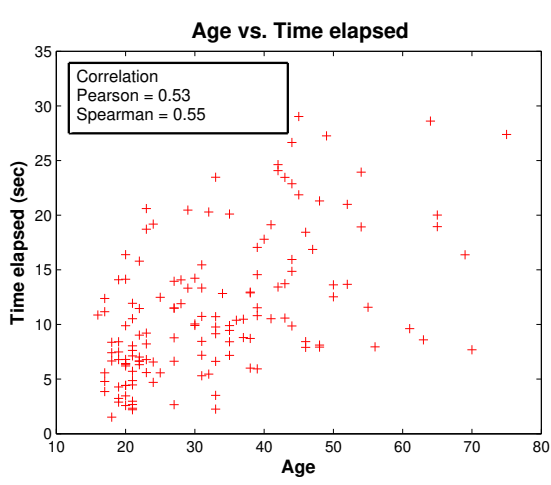

(a)

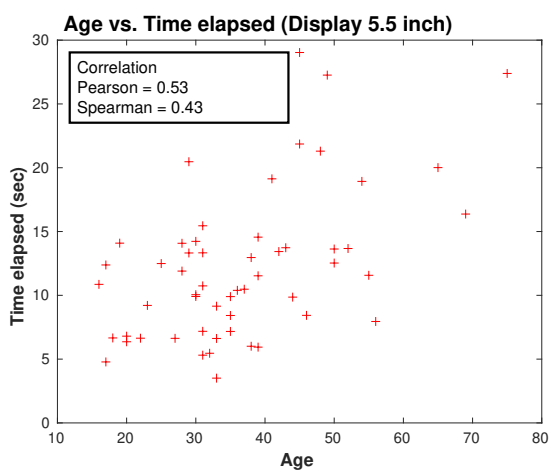

(b)

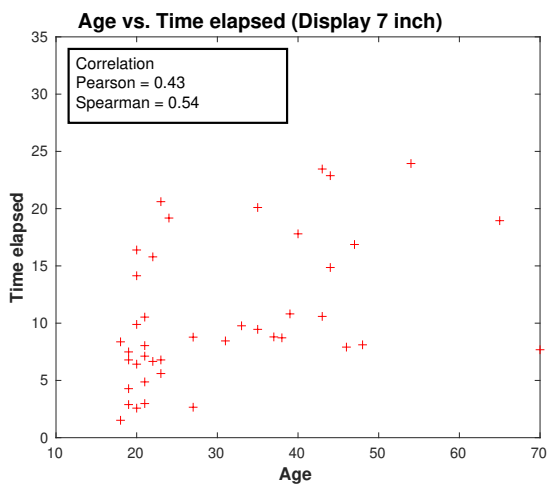

(c)

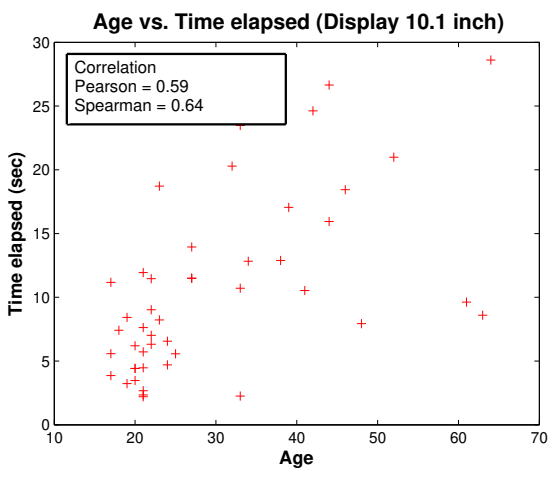

(d)

Figure 8. Pearson's and Spearman's correlation coefficients: correlation between age and volunteer performance in accomplishing tasks of experiments.

certain correlation between the age factor and the volunteer performance, although this correlation is not extremely high when we considered the data from all volunteers in our analysis. To verify if the display size is still more relevant than the age factor, the correlation coefficients were calculated considering separately the data of the volunteers who used the prototype with the 5.5, 7 and 10.1 inch displays. Figure 8 shows the results of this analysis.

The results showed that the correlation between age factor and volunteer performance increased only when the use of a larger display was considered (Figures $8 \mathrm{~b}, 8 \mathrm{c}$ and $8 \mathrm{~d}$ ). There was greater variation in the Spearman coefficient when the device had a 10.1 inch display (Figure 8d). These results indicate that, although age is an important factor, the display size of the device still has to be considered more relevant.

\section{CONCLUSION}

AR applications that are executed on handheld devices are becoming more common. A major challenge in developing applications in the area is to enable user-friendly visualization and interaction with the system, even if handheld devices have small displays compared to laptops and desktop computers.

Since the touch on the screen is the most common form of interaction in AR handheld applications, the present work has used subjective experiments based on prototype execution in a controlled environment to identify the impact of the type of device on user performance.

Regarding our hypothesis $\mathrm{H} 0$, results show that although some volunteers have stated that ergonomically smaller devices are easier to handle, average performance has gradually improved as the device and its display has been increased, even when the weight of the device has been increased. The user performance is statistically significant when we compared the data from the volunteers that used a 5.5 inch display with the data from the volunteers that used a 10.1 inch display.

Related to the hypothesis H1, we showed that there is no correlation between the height of the users and their ability to perform the task more efficiently. In addiction, the angle at which the user views the marker in the display has less influence than type of device. On the other hand, the volunteers habituated to use mobile games or AR applications have taken advantage of the device with larger displays.

The results also showed that there is a certain correlation between the age and the performance of a user. This correlation is not extremely high when we considered the data from all volunteers. However, when we grouped the data by the type of device this correlation is larger.

We concluded that new users are not able to improve their performance when the device is larger. Advanced users, on the other hand, presented improvement in the performance in the accomplishment of the assigned task. The age of the participant was also a good indicator of the user performance, since it may include other factors such as experience with technology, handling of mobile devices etc. However, the type of device is even more relevant.

The results obtained from this work may help developers to indicate to their users (or a group of users) when a type of device can affect their performance in an AR application. 
For example, when the AR application is a game in which the interaction is performed by touch on the screen, users are motivated to give their best performance. In this case, developers could recommend the use of large screens. On the other hand, there are applications where the interaction only aims to use a menu. Applications as "NYTimes - Latest News" or "Blippar" (see Tab I) do not encourage users to improve their performance. Information about possible performance improvement when larger screens are used are less relevant for these applications.

Future work include experiments without fiducial markers. Markless approaches may be used, such as the ARKit [23] (version 2 is available) and ARCore [24] frameworks, used in smartphones for mapping objects in the real environment.

\section{ACKNOWLEDGMENT}

The authors would like to thank the São Paulo Research Foundation (FAPESP), process number 2017/06619-3.

\section{REFERENCES}

[1] I. A. Rodello, S. R. R. Sanches, A. C. Sementille, and J. R. F. Brega, "Realidade misturada: Conceitos, ferramentas e aplicações," Revista Brasileira de Computação Aplicada, vol. 2, no. 2, pp. 2 - 16, 2010.

[2] B. F. Goldiez, A. M. Ahmad, and P. A. Hancock, "Effects of augmented reality display settings on human wayfinding performance," IEEE Transactions on Systems, Man and Cybernetics Part C: Applications and Reviews, vol. 37, no. 5, pp. 839-845, 2007.

[3] S. R. R. Sanches, D. M. Tokunaga, V. F. Silva, A. C. Sementille, and R. Tori, "Mutual occlusion between real and virtual elements in augmented reality based on fiducial markers," in 2012 IEEE Workshop on the Applications of Computer Vision (WACV), Jan 2012, pp. 49-54.

[4] J. Orlosky, T. Toyama, K. Kiyokawa, and D. Sonntag, "Modular: Eyecontrolled vision augmentations for head mounted displays," IEEE Transactions on Visualization and Computer Graphics, vol. 21, no. 11, pp. 1259-1268, Nov 2015.

[5] O. Bimber and R. Raskar, Spatial Augmented Reality: Merging Real and Virtual Worlds. Natick, MA, USA: A. K. Peters, Ltd., 2005.

[6] L. Ahmed, S. Hamdy, D. Hegazy, and T. El-Arif, "Interaction techniques in mobile augmented reality: State-of-the-art," in 2015 IEEE Seventh International Conference on Intelligent Computing and Information Systems (ICICIS), Dec 2015, pp. 424-433.

[7] S. Prasad, P. Kumar, and K. P. Sinha, "A wireless dynamic gesture user interface for hci using hand data glove," in 2014 Seventh International Conference on Contemporary Computing (IC3), Aug 2014, pp. 62-67.

[8] C.-Y. Lin and Y.-M. Chang, "Interactive augmented reality using scratch 2.0 to improve physical activities for children with developmental disabilities," Research in Developmental Disabilities, vol. 37, pp. 1 8, 2015.

[9] A. V. M. Moreira, V. V. Filho, and G. L. Ramalho, "Understanding mobile game success: a study of features related to acquisition, retention and monetization," SBC Journal on Interactive Systems, vol. 5, no. 2, pp. 2 - 13, 2014.

[10] "Pokémon GO," http://www.pokemongo.com/pt-pt/, 2016-12-12. [Online]. Available: http://www.pokemongo.com/pt-pt/

[11] M. Zamith, L. Valente, B. Feijó, M. Joselli, and E. Clua, "Parallel game architectures with tardiness policy and workload balance," SBC Journal on Interactive Systems, vol. 8, no. 1, pp. $74-86,2017$.

[12] L. Findlater and J. McGrenere, "Impact of screen size on performance, awareness, and user satisfaction with adaptive graphical user interfaces," in Proceedings of the SIGCHI Conference on Human Factors in Computing Systems, ser. CHI '08. New York, NY, USA: ACM, 2008, pp. 1247-1256. [Online]. Available: http://doi.acm.org/10.1145/1357054.1357249

[13] N. Maniar, E. Bennett, and D. Gal, "The effect that screen size has on video-based m-learning." in Fifth Annual IEEE International Conference on Pervasive Computing and Communications Workshops (PerComW'07), March 2007, pp. 145-148.

[14] E. Kruijff, J. E. Swan II, and S. Feiner, "Perceptual issues in augmented reality revisited." in ISMAR, vol. 9, 2010, pp. 3-12.
[15] A. Dey, G. Jarvis, C. Sandor, and G. Reitmayr, "Tablet versus phone: Depth perception in handheld augmented reality," in Mixed and Augmented Reality (ISMAR), 2012 IEEE International Symposium on. IEEE, 2012, pp. 187-196.

[16] D. Raptis, N. Tselios, J. Kjeldskov, and M. B. Skov, "Does size matter?: Investigating the impact of mobile phone screen size on users' perceived usability, effectiveness and efficiency." in Proceedings of the 15th International Conference on Human-computer Interaction with Mobile Devices and Services, ser. MobileHCI '13. New York, NY, USA: ACM, 2013, pp. 127-136. [Online]. Available: http://doi.acm.org/10.1145/2493190.2493204

[17] P. Ghazi and M. Glinz, "An exploratory study on user interaction challenges when handling interconnected requirements artifacts of various sizes," in 2016 IEEE 24th International Requirements Engineering Conference (RE), Sept 2016, pp. 76-85.

[18] D. Raneburger, R. Popp, D. Alonso-Rios, H. Kaind, and J. Falb, "A user study with guis tailored for smartphones and tablet pcs," in Systems, Man, and Cybernetics (SMC), 2013 IEEE International Conference on. IEEE, 2013, pp. 3727-3732.

[19] "Unity," https://unity3d.com, 2017-03-22. [Online]. Available: https://unity3d.com

[20] "Vuforia Developer Portal," https://developer.vuforia.com, 2017-03-22. [Online]. Available: https://developer.vuforia.com

[21] "Methodology for the subjective assessment of the quality of television pictures," ITU-R BT.500 https://www.itu.int/rec/R-REC-BT.500, 2009.

[22] S. R. R. Sanches, M. Oizumi, C. Oliveira, E. F. Damasceno, and A. C. Sementille, "Aspects of user profiles that can improve mobile augmented reality usage," in 2017 19th Symposium on Virtual and Augmented Reality (SVR), Nov 2017, pp. 236-242.

[23] Apple Inc. (2017) ARKit - Apple Developer. Accessed: December 27, 2017. [Online]. Available: https://developer.apple.com/arkit/

[24] Google LLC. (2018) Fundamental Concepts - ARCore Google Developers. Accessed: July 18, 2018. [Online]. Available: https://developers.google.com/ar/discover/concepts

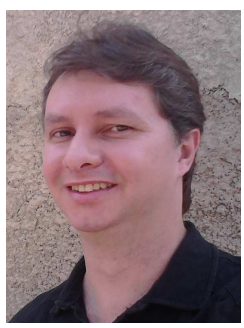

Silvio Ricardo Rodrigues Sanches received the B.Sc. and M.Sc. degree all from the Centro Universitário Eurípides de Marília (UNIVEM), Maríia, Brazil, in 2003 and 2007 respectively. He received the Ph.D. degree from the University of São Paulo (USP) at Escola Politécnica, São Paulo, Brazil, in 2013. Currently he is a Professor at Federal University of Technology - Paraná (UTFPR), Cornélio Procópio, PR, Brazil). His research interests include Augmented Reality and Computer Vision with special emphasis on user's perception.

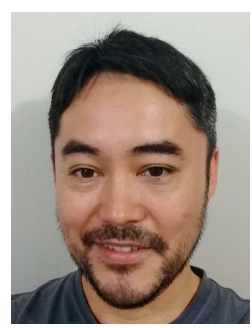

Marcio Adalberto Oizumi graduated in Systems Analysis from the Federal University of Technology - Paraná, Cornélio Procópio, Brazil, in 2017. His research interests include Augmented Reality, Image and Computer Vision with special emphasis on Human-Computer Interaction. 


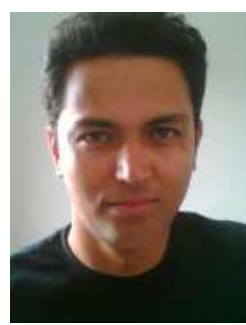

Claiton Oliveira received the B.Sc. degree in Computer Science from Londrina State University (UEL), Londrina, PR, Brazil, in 2005. He also received the M.Sc. degree in 2008 and the Ph.D. degree in 2012 both from the University of São Paulo (USP) at São Carlos School of Engineering (EESC), São Carlos, SP, Brazil. He is currently a Professor at Federal University of Technology - Paraná (UTFPR), Cornélio Procópio, PR, Brazil . His research interests include Image Processing and Computer Vision.

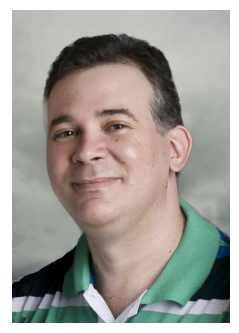

Antonio Carlos Sementille graduated in Computer Science from Universidade Estadual Paulista Júlio de Mesquita Filho - UNESP (1988). He has a M.Sc. degree in Computer Science from Universidade Federal de São Paulo, São Carlos (1994), a Ph.D. in Science (Computational Physics) from University of São Paulo (1999) and Livre Docência in Advanced Interfaces from UNESP (2010). Currently he is an Associate Professor at UNESP (Bauru). His research interests include Augmented Reality, Image Processing, Digital Television and Interactive Media.

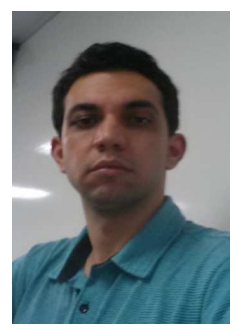

Cléber Gimenez Corrêa graduated in Data Processing from Faculty of Technology of São Paulo - FATEC, Brazil (2002). Master degree in Computer Science from University Center of Marília UNIVEM, Brazil (2008), and Ph.D. in Computer Engineering from Escola Politécnica, University of São Paulo - EPUSP, Brazil (2015). His research interests are haptic interface for medical training, haptic interaction objective perceptual assessment and software testing. Currently he is Professor at Federal University of Technology - Paraná. 